\title{
Molecular characterization of carbapenem- resistant Gram-negative bacilli clinical isolates in Algeria
}

This article was published in the following Dove Press journal: Infection and Drug Resistance

\author{
Nadjette Bourafa ${ }^{1-3}$ \\ Wafaa Chaalal ${ }^{1,4}$ \\ Sofiane Bakour' \\ Rym Lalaoui' \\ Nafissa Boutefnouchet ${ }^{2}$ \\ Seydina M Diene' \\ Jean-Marc Rolain' \\ 'Aix Marseille Universite, MEPHI, \\ IHU-Mediterranee Infection, Marseille, \\ France; ${ }^{2}$ Laboratoire de Microbiologie \\ et Biochimie Appliquée, Département \\ de Biochimie, Faculté des Sciences, \\ Université Badji Mokhtar Annaba, \\ Algeria; ${ }^{3}$ Département de Biologie, \\ Faculté des Sciences de la Nature \\ et de la Vie, Université Mohamed \\ Cherif Messaadia-Souk-ahras, Algeria; \\ ${ }^{4}$ Laboratoire de Microbiologie \\ Appliquée, Faculté des Sciences de la \\ Nature et de la Vie, Université d'Oran, \\ Es Senia, Oran, Algeria
}

Correspondence: Jean-Marc Rolain Aix Marseille Universite, MEPHI, IHUMediterranee Infection, 19-21 Boulevard Jean Moulin, 13005 Marseille, France Tel +33491324375

Fax +33491387772

Email jean-marc.rolain@univ-amu.fr
Objectives: The aims of this study were to investigate the occurrence of carbapenem-resistant Gram-negative bacilli (GNB) isolated from inpatients and outpatients in Algeria between July and September 2015, and to screen their resistance mechanisms and genetic relatedness.

Materials and methods: A total of 68 non-redundant isolates were identified using matrixassisted laser desorption/ionization time-of-flight (MALDI-TOF) antibiotic susceptibility testing was performed using disk diffusion and Etest methods. Carbapenemase activity was carried out using modified Carba NP test, EDTA assay, and the modified Hodge test. Molecular characterization of carbapenemases and extended-spectrum $\beta$-lactamase (ESBL) genes were detected by standard PCR and sequencing. Genotyping of carbapenem-resistant isolates was performed by multilocus sequence typing (MLST) analysis.

Results: Of the 68 GNB isolates, 13 (19\%) showed reduced susceptibility to carbapenems, including, four Klebsiella pneumoniae, one Escherichia coli, six Acinetobacter baumannii, and two Pseudomonas aeruginosa. bla ${ }_{\mathrm{OXA}-48}$ gene was detected in the five Enterobacteriaceae isolates, and $b l a_{\text {OXA-23 }}$ was identified in all A. baumannii isolates. OprD mutations were revealed in the two $P$. aeruginosa isolates. A total of 11 out of the 13 carbapenem-resistant GNB were detected in inpatients, and the two remaining strains were isolated from outpatients. Molecular typing showed the presence of four sequence types (STs) among the OXA-48-producing K. pneumoniae isolates: ST101, ST147, ST163, and ST2017. ST533 was identified for the OXA-48 producing E. coli isolate. All of the A. baumannii and P. aeruginosa were assigned to the international clonal lineages ST2 and ST654, respectively.

Conclusion: This study reports the first detection of the epidemic multidrug-resistant lineage, $K$. pneumoniae ST147 coproduced bla ${ }_{\mathrm{OXA}-48}$ and ESBL genes in Algeria and represents the first description of OXA-48-producing E. coli ST533 and K. pneumoniae ST163 and ST2017. In addition, this study describes for the first time the emergence of OXA-48-producing $E$. coli and $K$. pneumoniae in the community in Algeria, leading to major problems for managing microbial infections.

Keywords: Gram-negative bacilli, carbapenem resistance, epidemic lineages, Algeria

\section{Introduction}

Antibiotic resistance has increased dramatically over the last few years, and the incidence of carbapenemase-producing Gram-negative bacilli (GNB) is rising in many countries around the world. ${ }^{1}$ Carbapenems are considered as one of the last resorts for treating multidrug-resistant $\mathrm{GNB},{ }^{2-4}$ including ESBL-producing Enterobacteriaceae. ${ }^{5}$

Carbapenem resistance has been described in non-fermentative GNB (Acinetobacter baumannii and Pseudomonas aeruginosa) and in Enterobacteriaceae, ${ }^{2,6}$ mainly in Klebsiella pneumoniae compared to Escherichia coli or other Enterobacterial species. ${ }^{2,3}$ 
These GNB are major nosocomial pathogens ${ }^{7}$ and are the most common bacteria responsible for a wide range of community-acquired and nosocomial infections such as pneumonia, septicemia, peritonitis, meningitis, and urinary tract infections. ${ }^{4,7}$ Therefore, acquired carbapenemase-encoding genes constitute a real clinical concern for antimicrobial management, ${ }^{3}$ notably in that resistance to carbapenems may be coupled with resistance to other classes of antibiotics, such as aminoglycosides and fluoroquinolones. ${ }^{3}$

Carbapenem resistance in GNB can be mediated by carbapenem-hydrolyzing $\beta$-lactamases, including class $\mathrm{A}$ $\beta$-lactamases (KPC, IMI, and GES), class B metallo- $\beta$ lactamases (NDM, IMP, and VIM), and class D $\beta$-lactamases (OXA-48, OXA-23, OXA-24, and OXA-58). ${ }^{1,3,5,8}$ OXA $\beta$ lactamases were first described in $P$. aeruginosa; those carbapenemases have subsequently been reported in many other GNB, including A. baumannii and Enterobacteriaceae. ${ }^{3}$ The OXA-48 enzyme is the most frequently detected carbapenemase in Enterobacteriaceae., 3 This carbapenemase is not inhibited by clavulanic acid or ethylene diamine tetra-acetic acid (EDTA). ${ }^{3,4,6}$ Its spectrum of activity is substantial against penicillins, but it has low activity against third-generation cephalosporin, aztreonam, and carbapenems. ${ }^{3,6}$ The decreased permeability due to the loss or alteration of $o p r D$ porin in the outer membrane has been mainly described as linked to carbapenem resistance, especially in $P$. aeruginosa strains. ${ }^{2}$ Resistance mechanisms to imipenem in $P$. aeruginosa can also be related to the production of carbapenemases or to the presence of an efflux pump. ${ }^{9,10}$

In Algeria, the spread of carbapenem resistance in hospitalized patients has been mainly reported among $A$. baumannii, followed by $P$. aeruginosa, and very few cases have been reported among Enterobacteriaceae. However, until now, no information regarding the dissemination of carbapenem resistance among Algerian outpatients has been reported.

The objectives of this study were to investigate the occurrence of carbapenem-resistant GNB isolated from hospitalized and external patients in Algeria and to screen extended-spectrum $\beta$-lactamase (ESBL)-encoding genes and carbapenem resistance mechanisms in resistant isolates. The clonal relatedness of strains was assessed using multilocus sequencing typing (MLST).

\section{Materials and methods}

\section{Bacterial isolates and species} identification

A total of 68 non-redundant GNB isolated from hospitalized $(42.6 \%)$ and community $(57.3 \%)$ patients were recovered from the clinical microbiology laboratory at an Algerian hospital in Annaba city in North East Algeria, between July and September 2015. It was notable that patients from the community included in this study had not had a previous hospitalization or antibiotic treatment. The 68 clinical specimens were collected from 68 different patients.

Strains were identified using the API 20E and the API 20NE strips (bioMérieux, Marcy-l'Étoile, France), for the Enterobacteriaceae and the Non-Enterobacteriaceae isolates, respectively, and confirmed using matrix-assisted laser desorption/ionization time-of-flight (MALDI-TOF) mass spectrometry (MS) (Microflex ${ }^{\mathrm{TM}}$; Bruker Daltonic, Bremen, Germany) with flex control and biotyper 3.0 software (Bruker Daltonic).

\section{Antibacterial susceptibility testing}

Antimicrobial drug susceptibility was determined by the standard disc diffusion method on Mueller-Hinton agar as recommended by the Antibiogram Committee of the French Society for Microbiology/European Committee for Antimicrobial Susceptibility Testing (CA-SFM/EUCAST). ${ }^{11}$ The antimicrobial agents used were ticarcillin, ticarcillin/clavulanic acid, ceftriaxone, ceftazidime, cefepime, aztreonam, ertapenem, imipenem, gentamicin, amikacin, ciprofloxacin, fosfomycin, and trimethoprim-sulfamethoxazole (all from I2A, Montpellier, France). The minimum inhibitory concentrations (MICs) were determined using Etest strips (bioMérieux) for colistin in all strains and for imipenem in all carbapenem-intermediate or resistant isolates. Interpretations were made according to CA-SFM/EUCAST breakpoints.

\section{Phenotypic screening of carbapenemases}

Carbapenemase production was screened phenotypically for all strains exhibiting resistance or intermediate resistance to carbapenems using the modified Carba NP (MCNP) test, EDTA assay and the modified Hodge test (MHT) as previously described. ${ }^{7,12}$

\section{Molecular mechanisms of antibiotic resistance}

DNA extraction from all isolates showing non-susceptibility to carbapenems was performed using EZ1 DNA extraction kits (Qiagen NV, Venlo, the Netherlands) with the EZ1 Advanced XL biorobot according to the manufacturer's instructions.

Real-time PCR and standard PCR were performed to screen for the presence of carbapenem hydrolyzing enzymeencoding genes: $b l a_{\text {OXA-23 }}, b l a_{\text {OXA-24 }}, b l a_{\text {OXA-58 }}, b l a_{\text {OXA-48 }}, b l a_{\text {KPC }}$, 
$b l a_{\mathrm{IMP}}, b l a_{\mathrm{VIM}}$, and $b l a_{\mathrm{NDM}}$ genes, ${ }^{2}$ and ESBL genes $\left(b l a_{\mathrm{CTX}-\mathrm{M}}\right.$, bla $a_{\mathrm{TEM}}, b l a_{\mathrm{SHV}}$ bla $a_{\mathrm{GES}}, b l a_{\mathrm{VEB}}$, and $\left.b l a_{\mathrm{PER}}\right) .{ }^{13}$ The positive PCR products were purified and sequenced using the Big Dye terminator chemistry on an ABI 3130XL automated sequencer (Thermo Fisher Scientific, Waltham, MA, USA). OprD mutations were investigated on imipenem-resistant $P$. aeruginosa isolates. The obtained sequences were analyzed using Codon Code Aligner software and then examined using the BlastN and BlastP compared against the NCBI database (www. ncbi.nlm.nih.gov) and ARG-ANNOT (Antibiotic Resistance Gene-ANNOTation) (http://en.mediterranee-infection.com/ article.php?laref=283\&titre=arg-annot-).

\section{Molecular typing by MLST}

Molecular typing of carbapenem-resistant isolates was carried out by MLST, as described on the E. coli MLST Database (http://mlst.warwick.ac.uk/mlst/dbs/Ecoli) for $E$. coli, for $P$. aeruginosa (https://pubmlst.org/paeruginosa/), and according to the Pasteur schemes available at Institute Pasteur's MLST Web site (bigsdb.web.pasteur.fr) for $K$. pneumoniae and A. baumannii.

\section{Statistical analysis}

Chi-square tests and Fisher's exact tests were performed using two-sided comparisons to establish the statistical difference in levels of antibiotic resistance between hospitalized and external patients. Statistical significance was set at $P<0.05$.

\section{Results}

\section{Bacterial strain identification}

Identification of GNB using conventional microbiological tests and MALDI-TOF MS showed that Enterobacteriaceae represent $69.1 \%$ of the strains $(\mathrm{n}=47)$, including $E$. coli $(57.4 \% ; n=27)$ and $K$. pneumoniae $(42.5 \% ; n=20)$. Nonfermentative isolates represent $30.9 \%$ of the strains $(n=21)$, including A. baumannii $(57.1 \% ; \mathrm{n}=12)$ and $P$. aeruginosa $(42.8 \% ; n=9)$. The strains were isolated from various clinical specimens, including urine $(51.5 \%)$, wounds $(26.5 \%)$, bloodstream infections $(20.6 \%)$, and cerebrospinal fluid (1.5\%).

\section{Antimicrobial susceptibility}

Antimicrobial resistance patterns according to species and patient types are shown in Table 1. The results showed that most of the isolates were resistant to ticarcillin and ticarcillin-clavulanic acid, and a high resistance level to ciprofloxacin and trimethoprim/sulfamethoxazole was observed. Cephalosporins and gentamicin exhibited high activity against $E$. coli strains compared to other GNB isolates. Amikacin and colistin were found to be the most active on the isolates tested. Carbapenem activity showed that one E. coli (3.7\%) and four K. pneumoniae isolates (20\%) exhibited resistance to ertapenem, with two of them also being intermediate to imipenem with MICs of $3 \mu \mathrm{g} /$ $\mathrm{mL}$. Six A. baumannii and two P. aeruginosa isolates were resistant to imipenem with MICs $>32 \mu \mathrm{g} / \mathrm{mL}$. On the other hand, we found that strains isolated from hospitalized patients were significantly more resistant than those of external origin $(P<0.05)$ for most of the antibiotics tested (Table 1).

\section{Phenotypic screening of carbapenemases}

Phenotypic detection of carbapenemases showed that EDTAantibiotic disc synergy test was negative for all carbapenem-resistant strains, which indicates the absence of metallo- $\beta$-lactamase production. The MCNP test and modified Hodge assay were found positive for all isolates resistant to carbapenem including K. pneumoniae $(\mathrm{n}=4)$, E. coli $(\mathrm{n}=1)$, and A. baumannii $(\mathrm{n}=6)$ except for the imipenem-resistant $P$. aeruginosa isolates $(\mathrm{n}=2)$.

\section{Molecular mechanisms of antibiotic resistance}

Molecular characterization of acquired carbapenemaseencoding genes showed the presence of the bla $a_{\text {OXA-48 }}$ gene in the four $K$. pneumoniae isolates and in one $E$. coli isolate resistant to carbapenem. All imipenem-resistant $A$. baumannii carried the bla $a_{\text {OXA-23 }}$ gene. OprD gene mutations were detected in the two imipenem-resistant $P$. aeruginosa isolates, using as reference that of the PAO1 reference strain. OprD gene mutations had several polymorphism types (deletion, substitution, and insertion) leading to the premature stop codon "TGA" on the OprD protein for the $P$. aeruginosa strain $\mathrm{S} 1$, and the premature stop codon TAG for the $P$. aeruginosa strain S2 (Table 2). The first mutations in the oprD genes are the substitution of $\mathrm{T}$ to $\mathrm{C}$ in the nucleotide number 243 for the strain (S1), and G to $A$ in the nucleotide number 220 for the strain (S2). None of the isolates were positive for $b l a_{\text {OXA-24 }}, b l a_{\text {OXA-58 }}, b l a_{\text {IMP' }}$ bla $_{\mathrm{KPC}}$, bla $_{\mathrm{VIM}}$, or bla $a_{\mathrm{NDM}}$ genes. All OXA-48-producing Enterobacteriaceae isolates from hospital origin showed co-resistance to most classes of the antimicrobial agents tested compared to isolates from external origin. On the other hand, we found that $18.2 \%$ of carbapenem-resistant isolates (one K. pneumoniae and one E. coli) were identified 
Table I Antimicrobial resistance patterns of Gram-negative bacilli clinical isolates according to species (A) and patient types (B)

\begin{tabular}{|c|c|c|c|c|c|c|c|}
\hline \multirow[t]{4}{*}{ Antibiotics } & \multicolumn{4}{|l|}{ (A) } & \multicolumn{3}{|l|}{ (B) } \\
\hline & \multicolumn{4}{|c|}{ Resistance pattern (\%) } & \multicolumn{2}{|c|}{ Patient types $^{\mathrm{a}}$} & \multirow[t]{3}{*}{$P$ values ${ }^{b}$} \\
\hline & \multicolumn{2}{|c|}{ Enterobacteriaceae $(n=47)$} & \multicolumn{2}{|c|}{ Non-Enterobacteriaceae $(n=2 I)$} & \multirow{2}{*}{$\begin{array}{l}\text { Inpatients } \\
(n=29)\end{array}$} & \multirow{2}{*}{$\begin{array}{l}\text { Outpatients } \\
(n=39)\end{array}$} & \\
\hline & $\begin{array}{l}\text { Escherichia } \\
\text { coli } \\
(\mathrm{n}=27)\end{array}$ & $\begin{array}{l}\text { Klebsiella } \\
\text { pneumoniae } \\
(n=20)\end{array}$ & $\begin{array}{l}\text { Pseudomonas } \\
\text { aeruginosa } \\
(\mathrm{n}=9)\end{array}$ & $\begin{array}{l}\text { Acinetobacter } \\
\text { baumannii } \\
(n=\mid 2)\end{array}$ & & & \\
\hline TIC & $25(92.6)$ & $19(95)$ & 8 (88.9) & II (9I.7) & 29 & 34 & 0.1 \\
\hline TCC & $24(88.9)$ & $15(75)$ & 8 (88.9) & II (9I.7) & 28 & 30 & 0.04 \\
\hline CAZ & $9(33.3)$ & $17(85)$ & 7 (77.8) & II (9I.7) & 23 & 21 & 0.02 \\
\hline CRO & $9(33.3)$ & $16(80)$ & Nd & Nd & $18^{c}$ & $7^{c}$ & $<10^{-3 c}$ \\
\hline FEP & $5(18.5)$ & $16(80)$ & $5(55.5)$ & $9(75)$ & 21 & 14 & 0.002 \\
\hline ATM & $7(26)$ & $15(75)$ & $5(55.5)$ & $9(75)$ & 21 & 15 & 0.005 \\
\hline ETP & I (3.7) & $4(20)$ & Nd & Nd & $3^{c}$ & $2^{c}$ & $0.5^{c}$ \\
\hline IPM & 0 & $2(10)$ & $2(22.2)$ & $6(50)$ & 10 & 0 & $<10^{-3}$ \\
\hline GN & 9 (33.3) & $14(70)$ & $5(55.5)$ & $8(66.7)$ & 18 & 18 & 0.2 \\
\hline AMK & 0 & I (5) & 0 & $4(33.3)$ & 5 & 0 & 0.02 \\
\hline CIP & I5 (55.5) & $14(70)$ & 7 (77.8) & II (9I.7) & 24 & 23 & 0.03 \\
\hline FOF & 0 & $12(60)$ & 7 (77.8) & $\mathrm{Nd}$ & $17^{d}$ & $2^{d}$ & $<10^{-3 \mathrm{~d}}$ \\
\hline SXT & $23(85.2)$ & $14(70)$ & $\mathrm{Nd}$ & $12(100)$ & $26^{e}$ & $23^{e}$ & $0.02^{\mathrm{e}}$ \\
\hline $\mathrm{CT}$ & 0 & 0 & 0 & 0 & 0 & 0 & $\mathrm{Nd}$ \\
\hline
\end{tabular}

Notes: ${ }^{\mathrm{G}} \mathrm{GNB}$ strains were isolated from 29 inpatients $(E$. coli $\mathrm{n}=8, K$. pneumoniae $\mathrm{n}=10, P$. aeruginosa $\mathrm{n}=3$, and $A$. baumannii $\mathrm{n}=8)$ and 39 outpatients $(E$. coli $\mathrm{n}=19, \mathrm{~K}$. pneumoniae $n=10, P$. aeruginosa $n=6$, and $A$. baumannii $n=4$ ). 'Statistical tests were performed using two-sided Pearson $\times 2$ or Fisher's exact test, as appropriate, comparing the levels of antibiotic resistance between hospitalized and external patients. ${ }^{C} P$-values for CRO and ETP were evaluated only for Enterobacteriaceae isolates. ${ }^{d} P$-values for FOF were evaluated only for Enterobacteriaceae and $P$. aeruginosa strains. ${ }^{e} P$-values for SXT were evaluated only for Enterobacteriaceae and $A$. baumannii isolates.

Abbreviations: TIC, ticarcillin; TCC, ticarcillin/clavulanic acid; CAZ, ceftazidime; CRO, ceftriaxone; FEP, cefepime; ATM, aztreonam; ETP, ertapenem; IPM, imipenem; GN, gentamicin; AMK, amikacin; CIP, ciprofloxacin; FOF, fosfomycin; SXT, trimethoprim/sulfamethoxazole; CT, colistin; Nd, not determined.

in outpatient strains (Table 2). The molecular analysis of $\beta$-lactamases encoding genes for carbapenem-resistant isolates is presented in Table 2 .

\section{Molecular typing by MLST}

Genotyping of carbapenem-resistant Enterobacteriaceae isolates revealed that the OXA-48-producing $E$. coli isolate belonged to sequence type (ST)533, and the four OXA48-producing $K$. pneumoniae isolates belonged to four distinct STs, including ST163, ST2017, and the international high-risk resistant lineages ST101 and ST147. MLST results for carbapenem-resistant non-fermentative isolates showed that all of $A$. baumannii and $P$. aeruginosa strains were assigned to the international clonal lineages ST2 and ST654, respectively (Table 2).

\section{Discussion}

Increasing drug resistance causes a real problem in therapeutic choices, especially with the rise of carbapenem resistance among GNB isolates, which has emerged and been reported in many countries. ${ }^{1,3,4}$ In Algeria, carbapenem-resistant Enterobacteriaceae have rarely been reported compared to non-fermentative pathogens. In our epidemiological survey, we observed an increased frequency of infection with
OXA-48 carbapenemase-producing E. coli (3.7\%) and $K$. pneumoniae (20\%) isolates. The first description of OXA48-producing E. coli in Algeria was reported in 2014 with the prevalence of $1.1 \%$ among the collection of 448 isolates. ${ }^{8}$ In addition, Agabou et al ${ }^{14}$ reported in 2016 the detection of an OXA-48-producing E. coli strain isolated from a patient in the Constantine region. OXA-48-producing $E$. coli strains were also reported in numerous countries, including France, Belgium, Germany, Italy, Spain, India, Turkey, Lebanon, and Senegal. ${ }^{6}$ OXA-48-producing K. pneumoniae was first reported in Turkey in $2001 .^{3,4,15}$ It has since been extensively identified in Turkey and many regions of the world including neighboring countries such as Morocco, Tunisia, and Libya, ${ }^{4,5,15}$ and in Mediterranean countries (France, Spain, Italy, Slovenia, Greece, Egypt, and Lebanon) ${ }^{3,4,6,8}$ The occurrence of OXA-48-producing $K$. pneumoniae has also been reported in Senegal, Saudi Arabia, India, Ireland, Switzerland, and Russia. ${ }^{6}$ Recently, in Algeria, a few clinical cases of OXA-48-producing K. pneumoniae have been described: in Algiers (one isolated case, in 2014) ${ }^{16}$ in Constantine (three isolated cases, in 2015), ${ }^{1}$ in Guelma (two isolated cases, in 2014), ${ }^{17}$ in Ouargla (one isolated case recovered between 2014 and 2015), ${ }^{13}$ in Batna (seven isolated strains collected between 2014 and 2015), ${ }^{18}$ and two strains isolated from the 


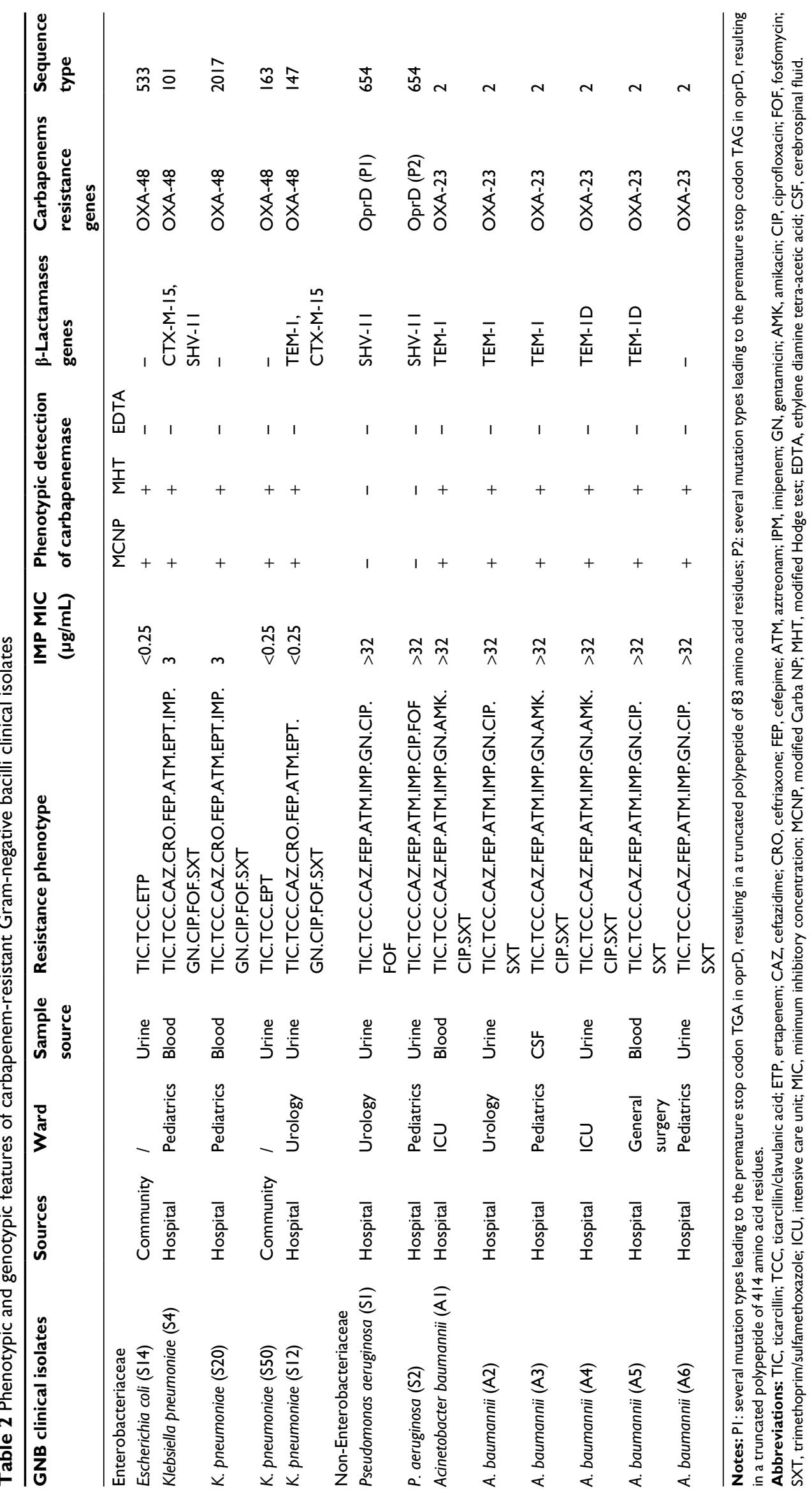


collection of 55 isolates collected between 2013 and 2015 from hospitalized patients in the cities of Annaba (one isolate) and Skikda (one isolate). ${ }^{2}$ However, it is important to note that the Enterobacteriaceae family- and also non-fermentative bacilli-producing carbapenemase have always been associated with hospitalized patients. The increased prevalence of carbapenemase producers in the hospital setting occurred as a result of many factors, including antibiotic selection pressure, ${ }^{6}$ the horizontal transfer of plasmids containing carbapenemase genes between different Enterobacteriaceae species, ${ }^{6}$ and the spread of clones harboring these genes among inpatients through hand contact with health care personnel. However, it is worth noting that in this study, $18.2 \%$ of carbapenemases detected (two out of the 11) were identified from outpatient strains (one in E. coli and one in K. pneumoniae isolates). Given that the outpatients included in our investigation had not previously used antibiotics, this suggests that resistance could be acquired via contact with animals or transmitted to humans through the food chain. ${ }^{14,19}$ Therefore, our document represents the first report describing $E$. coli and $K$. pneumoniae isolates harboring the $b l a_{\text {OXA-48 }}$ carbapenemase gene from the community in Algeria. Notwithstanding, OXA-48 carbapenemase producers of community origin have been reported in Morocco and Switzerland. ${ }^{20,21}$ The emergence of carbapenemase-producing strains in the community represents a major epidemiological and therapeutic problem. Furthermore, the emergence of carbapenem-resistant Enterobacteriaceae producing OXA-48 of animal origin and from environmental samples has been reported in Algeria ${ }^{17,22}$ and in other countries..$^{23,24}$

In this study, the identification of OXA-48-producing $E$. coli and $K$. pneumoniae from external patients highlights the wide dissemination of the bla $a_{\text {OXA-48 }}$ gene in Algeria. On the other hand, it should be noted that these two OXA-48 carbapenemase producers from the community were resistant to ertapenem but susceptible to imipenem. Indeed, comparing the resistance pattern of carbapenemase-producing strains of hospital origin with those of external origin, we found that external origin strains were more sensitive and also were negative for ESBL production (Table 2). That is why we propose to use the ertapenem antibiotic for detecting OXA-48 carbapenemase, particularly in non-multidrug resistant strains.

In our investigation, we report the first identification of the epidemic multidrug-resistant lineage $K$. pneumoniae ST147, co-producing ESBL and the $b l a_{\text {OXA-48 }}$ gene from a hospital setting in Algeria. Also, the present study represents the second report of the internationally successful lineage ST101-producing OXA-48 in Algeria. The first identification of this ST was very recently reported in an outbreak of OXA- 48-producing K. pneumoniae in Batna University Hospital, Algeria. ${ }^{18}$ Interestingly, these epidemic strains carrying $b l a_{\text {OXA-48 }}$ beta-lactamase have been involved in several outbreaks in North African countries and also in some European regions. ${ }^{15,25}$ We note that strains belonging to these lineages often harbor resistance determinants for different classes of antibiotics, such as $\beta$-lactam, quinolone, and carbapenem..$^{25,26}$ In addition, our report describes the first detection of OXA-48 carbapenemase-producing E. coli ST533 and K. pneumoniae ST163 and ST2017 in Algeria.

E. coli ST533 and K. pneumoniae ST163 of clinical origin have been described in Korea and China, respectively. ${ }^{27,28}$ However, E. coli ST533 had previously been detected in the gut of chickens in $\mathrm{China}^{29}$ and in uropathogenic strains isolated from dogs in Switzerland. ${ }^{30}$ To date, there are no reports describing K. pneumoniae ST2017. For GNB nonfermentative isolates, carbapenemase-encoding genes were identified only among $A$. baumannii isolates. The resistance of $A$. baumannii to carbapenem is mostly mediated by OXA23 carbapenemase. ${ }^{31}$ This oxacillinase has been extensively reported in numerous countries in the world, ${ }^{3,7,15}$ including Algeria, ${ }^{7,15,31}$ and was identified in all A. baumannii isolates resistant to imipenem included in this study. OXA-23 carbapenemase has thus become the dominant and endemic gene of carbapenem-resistant A. baumannii in Algerian hospitals. As reported, $A$. baumannii OXA-23 producers are generally multiresistant, ${ }^{32,33}$ similar to those found in our survey. MLST analysis showed that all $A$. baumannii OXA23 producers belonged to the international clone ST2, which has a wide geographical distribution. In addition, ST2 was the predominant clone detected in various clinical and environmental specimens, in Algeria, ${ }^{7,13}$ and in other countries. ${ }^{34,35}$ In this study, the molecular investigation of the carbapenems resistance mechanism in $P$. aeruginosa isolates revealed the chromosomic mutations in the $\operatorname{opr} D$ gene. The alteration of the oprD gene was the main mechanism for imipenem resistance in $P$. aeruginosa clinical strains, as previously described in Algeria ${ }^{2,9}$ and in other countries. ${ }^{10,36}$ On the other hand, we found that imipenem-resistant $P$. aeruginosa isolates displayed multidrug resistance profiles and also harboring of the ESBL gene. To our knowledge, ESBL genes have never been detected in imipenem-resistant $P$. aeruginosa strains in Algeria. Moreover, these isolates were assigned to the successful international clone ST654, usually associated with a multidrug-resistant phenotype, ${ }^{37}$ and this clone has been previously reported in Algeria, among imipenem-resistant $P$. aeruginosa clinical and surface strains that had mutational inactivation of oprD gene. In addition, the clone ST654 associated with KPC and VIM-producing P. aeruginosa has 
been described in Argentina and in the United Kingdom, respectively. ${ }^{37}$

\section{Conclusion}

This study represents the first report of epidemic multidrugresistant lineage $K$. pneumoniae ST147 co-producing $b l a_{\text {OXA-48 }}$ and ESBL genes in Algeria and describes the first detection of OXA-48-producing E. coli ST533 and K. pneumoniae ST163 and ST2017. Also, this study represents the first description of ESBL-producing imipenem-resistant $P$. aeruginosa strains in Algeria. In addition, this study showed that carbapenem-resistant GNB are propagated in this country. E. coli and K. pneumoniae isolates harboring bla $a_{\text {OXA-48 }}$ carbapenemase are emerging in the community, which is very worrying. Therefore, we highlight the need to establish monitoring systems in order to prevent a much wider dissemination of carbapenemase producers in Algeria.

\section{Acknowledgments}

We are very grateful to Linda Hadjadj for technical assistance and very grateful to TradOnline for the English proofreading of the paper.

This work was supported by the French Government under the "Investments for the Future" program managed by the National Agency for Research (ANR), MéditerranéeInfection 10-IAHU-03.

\section{Disclosure}

The authors report no conflicts of interest in this work.

\section{References}

1. Cuzon G, Bentchouala C, Vogel A, et al. First outbreak of OXA48-positive carbapenem-resistant Klebsiella pneumoniae isolates in Constantine, Algeria. Int J Antimicrob Agents. 2015;46(6):725-727.

2. Mellouk FZ, Bakour S, Meradji S, et al. First detection of VIM-4-producing Pseudomonas aeruginosa and OXA-48-producing Klebsiella pneumoniae in Northeastern (Annaba, Skikda) Algeria. Microb Drug Resist. 2017;23(3):335-344.

3. Djahmi N, Dunyach-Remy C, Pantel A, Dekhil M, Sotto A, Lavigne JP. Epidemiology of carbapenemase-producing Enterobacteriaceae and Acinetobacter baumannii in Mediterranean countries. Biomed Res Int. 2014;2014:305784.

4. Nordmann P, Naas T, Poirel L. Global spread of carbapenemase-producing Enterobacteriaceae. Emerg Infect Dis. 2011;17(10):1791-1798.

5. Hays C, Benouda A, Poirel L, Elouennass M, Nordmann P. Nosocomial occurrence of OXA-48-producing enterobacterial isolates in a Moroccan hospital. Int J Antimicrob Agents. 2012;39(6):545-547.

6. Poirel L, Potron A, Nordmann P. OXA-48-like carbapenemases: the phantom menace. J Antimicrob Chemother. 2012;67(6):1597-1606.

7. Bakour S, Olaitan AO, Ammari H, et al. Emergence of colistin- and carbapenem-resistant Acinetobacter baumannii ST2 clinical isolate in Algeria: first case report. Microb Drug Resist. 2015;21(3):279-285.

8. Agabou A, Pantel A, Ouchenane Z, et al. First description of OXA48-producing Escherichia coli and the pandemic clone ST131 from patients hospitalised at a military hospital in Algeria. Eur J Clin Microbiol Infect Dis. 2014;33(9):1641-1646.
9. Sefraoui I, Berrazeg M, Drissi M, Rolain JM. Molecular epidemiology of carbapenem-resistant Pseudomonas aeruginosa clinical strains isolated from western Algeria between 2009 and 2012. Microb Drug Resist. 2014;20(2):156-161.

10. Lee JY, Ko KS. OprD mutations and inactivation, expression of efflux pumps and AmpC, and metallo-beta-lactamases in carbapenem-resistant Pseudomonas aeruginosa isolates from South Korea. Int J Antimicrob Agents. 2012;40(2):168-172.

11. Antibiogram Committee of the French Society for Microbiology/European Committee for Antimicrobial Susceptibility Testing. CA-SFM/ Eucast. 2015- Version 1.0. Available from: http://www.sfm microbiologie.org/UserFiles/files/casfm/CASFM_EUCAST_V1_2015.pdf

12. Bakour S, Garcia V, Loucif L, et al. Rapid identification of carbapenemase-producing Enterobacteriaceae, Pseudomonas aeruginosa and Acinetobacter baumannii using a modified Carba NP test. New Microbes New Infect. 2015;7:89-93.

13. Yagoubat M, Ould El-Hadj-Khelil A, Malki A, et al. Genetic characterisation of carbapenem-resistant Gram-negative bacteria isolated from the University Hospital Mohamed Boudiaf in Ouargla, southern Algeria. J Glob Antimicrob Resist. 2017;8:55-59.

14. Agabou A, Lezzar N, Ouchenane Z, et al. Clonal relationship between human and avian ciprofloxacin-resistant Escherichia coli isolates in North-Eastern Algeria. Eur J Clin Microbiol Infect Dis. 2016;35(2): 227-234.

15. Mathlouthi N, Al-Bayssari C, Bakour S, et al. Prevalence and emergence of carbapenemases-producing Gram-negative bacteria in Mediterranean basin. Crit Rev Microbiol. 2017;43(1):43-61.

16. Aggoune N, Tali-Maamar H, Assaous F, Benamrouche N, Naim M, Rahal K. Emergence of plasmid mediated carbapenemase OXA-48 in a Klebsiella pneumoniae strain in Algeria. J Glob Antimicrob Resist. 2014;2(4):327-329.

17. Bouguenoun W, Bakour S, Bentorki AA, Al Bayssari C, Merad T, Rolain JM. Molecular epidemiology of environmental and clinical carbapenemase-producing Gram-negative bacilli from hospitals in Guelma, Algeria: multiple genetic lineages and first report of OXA-48 in Enterobacter cloacae. J Glob Antimicrob Resist. 2016;7:135-140.

18. Loucif L, Kassah LA, Saidi M, Messala A, Chelaghma W, Rolain JM. Outbreak of OXA-48-producing Klebsiella pneumoniae involving an ST 101 clone in Batna University Hospital, Algeria. Antimicrob Agents Chemother. 2016;60(12):7494-7497.

19. Jakobsen L, Kurbasic A, Skjøt-Rasmussen L, et al. Escherichia coli isolates from broiler chicken meat, broiler chickens, pork, and pigs share phylogroups and antimicrobial resistance with community-dwelling humans and patients with urinary tract infection. Foodborne Pathog Dis. 2010;7(5):537-547.

20. Zurfluh K, Nuesch-Inderbinen MT, Poirel L, Nordmann P, Hächler H. Emergence of Escherichia coli producing OXA-48 beta-lactamase in the community in Switzerland. Antimicrob Resist Infect Control. 2015;4:9.

21. Barguigua A, El Otmani F, Talmi M, Zerouali K, Timinouni M. Emergence of carbapenem-resistant Enterobacteriaceae isolates in the Moroccan community. Diagn Microbiol Infect Dis. 2012;73(3): 290-291.

22. Yousfi M, Touati A, Mairi A, et al. Emergence of carbapenemaseproducing Escherichia coli isolated from companion animals in Algeria. Microb Drug Resist. 2016;22(4):342-346.

23. Liu X, Thungrat K, Boothe DM. Occurrence of OXA- 48 carbapenemase and other beta-lactamase genes in ESBL-producing multidrug resistant Escherichia coli from dogs and cats in the United States, 2009-2013. Front Microbiol. 2016;7:1057.

24. Pantel A, Richaud-Morel B, Cazaban M, Bouziges N, Sotto A, Lavigne JP. Environmental persistence of OXA-48-producing Klebsiella pneumoniae in a French intensive care unit. Am J Infect Control. 2016; 44(3):366-368.

25. Lee CR, Lee JH, Park KS, Kim YB, Jeong BC, Lee SH. Global dissemination of carbapenemase-producing Klebsiella pneumoniae: epidemiology, genetic context, treatment options, and detection methods. Front Microbiol. 2016;7:895. 
26. Kocsis B, Kocsis E, Fontana R, Cornaglia C, Mazzariol A. Identification of blaLAP-(2) and qnrS1 genes in the internationally successful Klebsiella pneumoniae ST147 clone. J Med Microbiol. 2013;62(Pt 2): 269-273.

27. Lee SY, Park YJ, Yu JK, et al. Prevalence of acquired fosfomycin resistance among extended-spectrum beta-lactamase-producing Escherichia coli and Klebsiella pneumoniae clinical isolates in Korea and IS26composite transposon surrounding fosA3. J Antimicrob Chemother. 2012;67(12):2843-2847.

28. Luo Y, Wang Y, Ye L, Yang J. Molecular epidemiology and virulence factors of pyogenic liver abscess causing Klebsiella pneumoniae in China. Clin Microbiol Infect. 2014;20(11):O818-0824.

29. Chen Y, Sun J, Liao XP, et al. Impact of enrofloxacin and florfenicol therapy on the spread of OqxAB gene and intestinal microbiota in chickens. Vet Microbiol. 2016;192:1-9.

30. Huber H, Zweifel C, Wittenbrink MM, Stephan R. ESBL-producing uropathogenic Escherichia coli isolated from dogs and cats in Switzerland. Vet Microbiol. 2013;162(2-4):992-996.

31. Mesli E, Berrazeg M, Drissi M, Beckhoucha SN, Roulain JP. Prevalence of carbapenemase-encoding genes including New Delhi metallo-beta-lactamase in Acinetobacter species, Algeria. Int J Infect Dis. 2013;17(9):e739-e743.
32. Touati M, Diene SM, Racherache A, Dekhil M, Djahoudi A, Rolain JM. Emergence of blaOXA-23 and blaOXA-58 carbapenemase-encoding genes in multidrug-resistant Acinetobacter baumannii isolates from University Hospital of Annaba, Algeria. Int J Antimicrob Agents. 2012;40(1):89-91.

33. Viana GF, Zago MC, Moreira RR, et al. ISAba1/blaOXA-23: a serious obstacle to controlling the spread and treatment of Acinetobacter baumannii strains. Am J Infect Control. 2016;44(5):593-595.

34. Pailhoriès H, Belmonte $\mathrm{O}$, Kempf M, et al. Diversity of Acinetobacter baumannii strains isolated in humans, companion animals, and the environment in Reunion Island: an exploratory study. Int J Infect Dis. 2015; 37:64-69.

35. Bakour S, Alsharapy SA, Touati A, Rolain JM. Characterization of Acinetobacter baumannii clinical isolates carrying bla(OXA-23) carbapenemase and 16S rRNA methylase armA genes in Yemen. Microb Drug Resist. 2014;20(6):604-609.

36. Al Bayssari C, Diene SM, Loucif L, et al. Emergence of VIM-2 and IMP-15 carbapenemases and inactivation of oprD gene in carbapenemresistant Pseudomonas aeruginosa clinical isolates from Lebanon. Antimicrob Agents Chemother. 2014;58(8):4966-4970.

37. Zowawi HM, Ibrahim E, Syrmis MW, Wailan AM, AbdulWahab A, Paterson DL. PME-1-producing Pseudomonas aeruginosa in Qatar. Antimicrob Agents Chemother. 2015;59(6):3692-3693.
Infection and Drug Resistance

\section{Publish your work in this journal}

Infection and Drug Resistance is an international, peer-reviewed openaccess journal that focuses on the optimal treatment of infection (bacterial, fungal and viral) and the development and institution of preventive strategies to minimize the development and spread of resistance. The journal is specifically concerned with the epidemiology of antibiotic

\section{Dovepress}

resistance and the mechanisms of resistance development and diffusion in both hospitals and the community. The manuscript management system is completely online and includes a very quick and fair peerreview system, which is all easy to use. Visit http://www.dovepress.com/ testimonials.php to read real quotes from published authors. 